\title{
Ensayos con el control autónomo de sistemas aéreos no tripulados tipo cuadricóptero para su aplicación didáctica en la enseñanza aeronaútica
}

Omri Alberto Amaya Carias

\section{Resumen}

El propósito de este artículo es presentar los resultados de una investigación en el área del control de una aeronave no tripulada, siendo la orientación al área específica del uso en temas de didáctica educativa. Los usos de hardware y software específicos utilizados en esta investigación, ambos de código abierto, dan una oportunidad de desarrollo en el tema educativo en el campo aeronáutico, pretendiendo de esta manera utilizarlos como plataforma para enseñanza demostrativa. La metodología se explica en el artículo, buscando la posibilidad de explorar nuevas líneas de investigación indagando en el contexto hondureño temas trabajados en otros países o aportando nuevos temas o nuevas perspectivas de estudio. Se propone como problema de estudio el poco conocimiento en la Universidad Nacional Autónoma de Honduras (UNAH) acerca de las aeronaves no tripuladas y sus aplicaciones con equipamiento para distintas funciones con potencial para ser utilizados en contextos pedagógicos y para la experimentación en otros campos. Se concluye que este primer acercamiento al tema de vehículos aéreos no tripulados abre para el Departamento de Ciencias Aeronáuticas (DCA) un amplio campo de investigación desde el cual se pueden hacer importantes aportaciones para aplicaciones innovadoras necesarias y oportunas en la enseñanza, la investigación, el desarrollo económico y la recreación.

Palabras Clave: educación aeronáutica, aeronave no tripulada, drone, programación.

\section{Abstract}

The purpose of this article is to present the results of an investigation in the area of control of an unmanned aircraft, being the orientation to the specific area of use in educational didactic subjects. The specific hardware and software specifics used in this study, both of open source, give an opportunity to development in the educational field in aeronautics, intending in this way to use them as platform for demonstrative teaching. The methodology is explained in the article, looking for the possibility of exploring new lines of research by searching in the Honduran context topics worked on in other countries or contributing new topics or new study perspectives. It is proposed as a study problem the lack of knowledge in the Universidad Nacional Autónoma de Honduras (UNAH) of unmanned aircraft and their applications with equipment for different functions with potential to be used in pedagogical contexts and for experimentation in other fields. It is concluded that this first approach to the issue of aerial vehicles opens for Departamento de Ciencias Aeronáuticas (DCA) a wide field of research from which important contributions can be made for the necessary and timely innovative applications in teaching, research, economic development and recreation. 
Keywords: aeronautical education, unmanned aircraft, drone, programming.

Omri Alberto Amaya Carias Honduras, Tegucigalpa M.D.C., Ciudad Universitaria, email:omri.amaya@unah.edu.hn Máster en Ordenamiento y Gestión del Territorio, Departamento de Ciencias Aeronáuticas, Facultad de Ciencias Espaciales, Universidad Nacional Autónoma de Honduras,

Fecha de Recepción: 24 de julio de 2017 / Fecha de Aprobación: 11 de Agosto de 2017 


\section{INTRODUCCIÓN}

Actualmente existe una amplia variedad de aplicaciones para aeronaves no tripuladas, dependiendo de las capacidades intrínsecas de desempeño y del equipamiento con el que se pueden complementar en condición de plataforma, sus usos pueden ir desde el reconocimiento de áreas geográficas hasta el uso bélicomilitar, pasando por la detección de minerales, la cobertura de eventos sociales, tomas cinematográficas, controles policiales, manejo de bosques, apoyo de reportes noticiosos y hasta entregas a domicilio.

Un interés que atrae fuertemente la atención del (DCA) lo constituyen las diversas aplicaciones que pueden tener las aeronaves no tripuladas para fines didácticos. Como en otras condiciones y para otros fines lo podría hacer un túnel de viento, un "dron" puede mostrar en un ambiente experimental las cuatro fuerzas que propician el vuelo de cualquier aeronave, además sería un objeto de estudio adecuado para modelar maniobras de aeronaves en pleno vuelo, simular aterrizajes y despegues, mostrar a escala las distancias que deben mantener las aeronaves con respecto al suelo, con respecto a distintos obstáculos y con respecto a otras aeronaves, entre otros muchos ejercicios.

El proceso investigativo definió los antecedentes, presentando así la problemática a analizar, con sus objetivos y fases del proceso de investigación. Posteriormente se presentan los resultados de la investigación, El primero lo conforman las anotaciones de una entrevista y de un conjunto de pruebas realizadas con una persona experimentada en temas de drones que posee varios de ellos, incluyendo el software y los instrumentos de control remoto; de este primer producto se deriva la descripción detallada de un proceso de pruebas. En un segundo resultado se reportan las prácticas realizadas por el investigador en el ambiente virtual de un curso en línea denominado "Navigation for Flying Robots", lo relevante de estos ejercicios fue la elaboración de varias rutinas de maniobras en un lenguaje de programación que fueron probadas en dicha página. Por último se realizó una serie de prácticas para probar la vinculación entre el vehículo AR.Drone 2.0 y la computadora, a través del interfaz que vincula las dos partes, definido como Autoflight.

El artículo de esta investigación finaliza con los apartados de Bibliografía, que enuncia los documentos o referencias consultadas.

\section{DATOS Y METODOLOGÍA}

\section{II.1 DEFINICIÓN DEL PROBLEMA}

El presente trabajo propone el desarrollo y generación de un ambiente de robótica aplicada a aeronaves no tripuladas, investigación que viene a desarrollar un espacio necesario en Honduras, específicamente en la UNAH, dado el compromiso de dicha institución en el desarrollo e innovación tecnológico en el país. Por lo que la problemática a plantear es:

Dado que en la actualidad existen diferentes aplicaciones y usos de vehículos con la capacidad de desempeñar tareas cada vez más complejas, lo cual puede llevarse a cabo al incorporar cámaras y distintas clases de sensores a los prototipos para llevar a cabo misiones de reconocimiento, fotogrametría y vigilancia; se constata la necesidad de conocer los sistemas de control autónomo de las aeronaves no tripuladas. Dicho análisis se llevará a cabo con un vehículo con 
una configuración lo suficientemente estable durante el vuelo, para poder emplearlo posteriormente como referente a tomar en cuenta en la construcción de aeronaves; en este caso particular se hará uso del software Autoflight en procesos de práctica y simulación en el drone AR. Drone 2.0. como punto de inicio.

\section{II.2 Los Datos}

La presente investigación utilizará prevalentemente datos cualitativos, relacionados con secuencias y tipos de movimientos realizados por el vehículo aéreo no tripulado, la obtención de los datos para la manipulación de la variable se fundamentará en: el programa Autoflight, el lenguaje Python, el AR. Drone 2.0 y Windows 7 de 64 bits. Por todo lo anterior se definen:

- Variable dependiente: modificaciones la secuencia de comandos Python a ser utilizados en Autoscript del programa Autoflight de acuerdo a la precisión del drone en las prácticas realizadas respondiendo a órdenes establecidas a través de dichos comandos $y$,

- Variable independiente: Diseño y características del AR. Drone 2.0.

\section{II.2.1 Inicios de la Aviación No Tripulada}

La aviación no tripulada tuvo sus comienzos en los modelos construidos y volados por inventores como Cayley, Stringfellow, Du Tempel y otros pioneros de la aviación, que fueron previos a sus propios intentos de desarrollar aeronaves tripuladas a lo largo de la primera mitad del siglo XIX. Estos modelos sirvieron como bancos de pruebas tecnológicos para el posterior desarrollo de modelos de mayor tamaño con piloto a bordo y, en este sentido, fueron los precursores de la aviación tripulada.

El termino vehículo aéreo no tripulado (Unmanned Aerial Vehicle, UAV) se hizo común en los años 90 para describir a las aeronaves robóticas y reemplazó el término "vehículo aéreo pilotado remotamente" (Remotely Piloted Vehicle, RPV), el cual fue utilizado durante la guerra de Vietnam y posteriormente. El documento "Joint Publication 1-02, Deparment of Defense Dictionary" editado por el Ministerio de Defensa de los Estados Unidos define UAV como: "Un vehículo aéreo motorizado que no lleva a bordo a un operador humano, utiliza las fuerzas aerodinámicas para generar la sustentación, puede volar autónomamente o ser tripulado de forma remota, que puede ser fungible o recuperable, y que puede transportar una carga útil letal o no. No se consideran UAV a los misiles balísticos o semibalisticos, misiles crucero y proyectiles de artillería".

Merece la pena prestar atención al acrónimo RPAS (Sistemas de Aeronaves Pilotadas a Distancia por sus siglas en inglés). En el año 2011 la Organización de Aviación Civil Internacional (OACI), organismo especializado de las Naciones Unidas para la aviación civil y del cual Honduras forma parte al haber suscrito el Convenio de Chicago en 1944, publicó su Circular 328 en la cual por primera vez reconoce a las aeronaves no tripuladas como aeronaves, con todo lo que ello trae consigo, y de entre todas las posibles tipologías escoge a las que se pilotan de manera remota para ser consideradas como aptas para la aviación civil, y no otros tipos (como podrían ser las autónomas). Así se han acuñado los términos que a continuación se detallan, y que tienen hoy en día una validez y aplicación internacional y casi única en todos los ámbitos. Estos términos son: 
- Aeronave pilotada remotamente (Remotely-Piloted Aircrarft, RPA): una aeronave en la que el piloto al mando no está a bordo;

- Sistema de aeronave pilotada remotamente (Reomtely-Piloted Aircraft System, RPAS): un conjunto de elementos configurables formados por un RPA, su estación de pilotaje remoto asociada (RPS-Remote Pilot Station), el sistema requerido de enlace de mando y control y cualquier otro elemento requerido en cualquier punto durante la operación de vuelo.

El resto de los acrónimos no definidos se corresponden con:

- UMA = Unmanned Aircraft;

- $A P V=$ Automatically Piloted Vehicle;

- UTA = Unmanned Tactical Aircraft;

- UCAV = Unmanned Combat Air Vehicle;

- ROA = Remotely Operated Aircraft.

Aunque las aeronaves no tripuladas han llevado a cabo operaciones comerciales tan variadas como agricultura de precisión o cinematografía, su uso militar se ha concentrado abrumadoramente en una aplicación: reconocimiento. De manera que el termino UAV o UAS (sistemas de aeronaves no tripulados; unmanned aerial system) se ha convertido virtualmente en un sinónimo de dicha aplicación (FENERCOM (2015)).

\section{II.2.2 Vehículos Aéreos No Tripulados.}

Definición: Un vehículo aéreo no tripulado (VANT, o UAV por sus siglas en inglés) es, como ya se indicó anteriormente, un aparato volador que no contiene un piloto en su interior, bien porque está siendo pilotado por control remoto o bien porque tiene en su programación todo lo necesario para llevar a cabo sus instrucciones sin intervención humana.

En un principio fueron diseñados para el ámbito militar, ya que se podía aprovechar al máximo el hecho de que carecía de un operador físicamente en la aeronave. Así, se podían efectuar operaciones de entrada en espacios aéreos peligrosos. Además, a parte de la ventaja de la seguridad, tenemos que el volumen necesario que necesita el aparato es mucho menor que el que necesita uno que deba albergar una persona, lo que repercute también en una mejor maniobrabilidad.

No obstante no todo es perfecto, y tantas ventajas no podían esconder menos desventajas. A medida que avanzamos en la era tecnológica damos más autonomía a las máquinas para quitarle trabajo a los humanos, lo que a priori parece perfecto, pero hay que tener en cuenta que aún tiene que pasar mucho tiempo hasta que una maquina pueda tener el mismo juicio que una persona. Si ya no es por ética, pensemos en la cantidad de hackers que hay por el mundo, y si pueden conseguir entrar a los lugares más seguros de internet, ¿muy difícil les será acceder al control de aeronaves no tripuladas militares? El VANT más antiguo del que se tiene constancia se desarrolló al final de la primera guerra mundial, y su utilidad era simplemente la de entrenar a los operarios de la artillería antiaérea.

No obstante, hasta el final del siglo XX no empiezan a aparecer DRONES que pueden operar con total autonomía controlados sólo por radio. 


\section{II.2.3 Tipos de Vehículos Aéreos No Tripulados}

En cuanto a la clasificación de los UAV podemos efectuarla conforme a tres criterios: su misión, su techo y alcance máximo y su morfología.

En cuanto a su misión distinguimos los siguientes tipos:

1. Blanco (Target): Como el primer UAV desarrollado al final de la primera guerra mundial, se utilizan para simular objetivos voladores.

2. Reconocimiento: Se encargan de obtener y enviar información militar, como por ejemplo imágenes aéreas de una base militar enemiga. En este tipo destacan los MUAV (Micro Vehículo Aéreo No Tripulado por sus siglas en inglés) en forma de avión o helicóptero.

3. Combate (UCAV): Los sustitutos de los pilotos de combate en misiones que pueden resultar muy peligrosas.

4. Logística: Diseñados simplemente para llevar carga.

5. Investigación y Desarrollo: Para probar los nuevos sistemas que están en investigación y desarrollo.

6. UAV comerciales y civiles: Los más conocidos y que se pueden conseguir fácilmente por internet. Para su uso civil y como entrenamiento.

En cuanto a su altura y alcance máximo en la Tabla 1 se pueden ver su clasificación. 
Tabla 1: Descripción de los Vehículos Aéreos No Tripulados seqún Clasificación de la OTAN en Septiembre del 2009 (Ministerio de Defensa de España (2009))

\begin{tabular}{|l|l|l|l|l|}
\hline Categoría & Acrónimo & $\begin{array}{l}\text { Alcance } \\
\text { máx. }(\mathrm{m})\end{array}$ & $\begin{array}{l}\text { Altitud } \\
\text { máx. }(\mathrm{m})\end{array}$ & $\begin{array}{l}\text { Carga } \\
\text { máx. }(\mathrm{kg})\end{array}$ \\
\hline Micro & Micro & $<10$ & 250 & $<5$ \\
\hline Mini & Mini & $<10$ & 300 & $<30$ \\
\hline $\begin{array}{l}\text { Alcance Cer- } \\
\text { cano }\end{array}$ & CR & $10-30$ & 3000 & 150 \\
\hline $\begin{array}{l}\text { Alcance cor- } \\
\text { to }\end{array}$ & SR & $30-70$ & 3000 & 200 \\
\hline $\begin{array}{l}\text { Alcance me- } \\
\text { dio }\end{array}$ & MR & $70-200$ & 5000 & 1250 \\
\hline Altitud baja & LADP & $>250$ & 9000 & 350 \\
\hline $\begin{array}{l}\text { Autonomía } \\
\text { media }\end{array}$ & MRE & $>500$ & 8000 & 1250 \\
\hline $\begin{array}{l}\text { Autonomía } \\
\text { alta Altitud } \\
\text { baja }\end{array}$ & LALE & $>500$ & 3000 & $>30$ \\
\hline $\begin{array}{l}\text { Autonomía } \\
\text { media Altitud } \\
\text { media }\end{array}$ & MALE & $>500$ & 14000 & 1500 \\
\hline $\begin{array}{l}\text { Autonomía } \\
\text { media Altitud } \\
\text { alta }\end{array}$ & HALE & $>2000$ & 20000 & 12000 \\
\hline Estratosférico & STRATO & $>2000$ & 30000 & ND \\
\hline $\begin{array}{l}\text { EXO- } \\
\text { Estratosférico }\end{array}$ & EXO & ND & $>30000$ & ND \\
\hline
\end{tabular}

En cuanto a su morfología distinguimos 5 tipos:

1. Ala Rotatoria: una morfología mundialmente conocida, con un rotor en la parte superior y uno de cola para compensar el par del rotor que lo haría girar sin dar vueltas. Tiene una alta maniobrabilidad y puede quedarse en vuelo estacionario y volar verticalmente.

2. Ala Fija: su morfología se presenta por dos alas horizontales en torno al fuselaje que lo dotan de la sustentación necesaria al ir a una determinada velocidad por diferencia de presiones. Puede ir a altas velocidades y llevar cargas elevadas, pero no tiene la posibilidad del vuelo estacionario ni tiene tanta maniobrabilidad como un helicóptero.

3. Dirigibles: mundialmente conocidos también durante la segunda guerra mundial, estos aparatos vuelan por un principio muy básico de diferencia de densidades.

4. Multirrotores: parecidos a los helicópteros, pero tienen varios rotores verticales en puntos equidistantes del centro horizontalmente, y variando las velocidades de giro de cada uno de los motores se consigue una maniobrabilidad sorprendente. Esto hace que sean las estrellas de los vuelos en interior, pero no son aptos para volar a grandes altitudes (EI UAV objeto de este proyecto se incluye en esta categoría). 
5. Hibrido: vehículo aéreo no tripulado UAV híbrido entre avión y helicóptero, lo que históricamente se ha dado a llamar convertible. Esta aeronave pretende aunar las ventajas de operación de las aeronaves de ala fija (aviones) con las de ala rotatoria (helicóptero).

\section{II.2.4 El Cuadricóptero: Parrot AR.Drone 2.0}

El Parrot AR.Drone es un vehículo aéreo no tripulado radio controlado de uso recreativo civil. Funciona propulsado por cuatro motores eléctrico en configuración cuadricóptero y es similar en su estructura básica y aerodinámica a otros modelos radio controlados, pero se diferencia de todos ellos en que cuenta con un microprocesador y una serie de sensores entre los cuales se incluyen dos cámaras que le permiten captar lo que ocurre a su alrededor, más un conector Wi-Fi Integrado que da la posibilidad de vincularse a dispositivos móviles personales con sistemas operativos IOS, Android o Linux. Es decir, es posible un control directo del cuadricóptero desde un dispositivo móvil, mientras se reciben a la vez imágenes y datos de telemetría que los sensores del AR.Drone receptan.

Este cuadricóptero se usa en universidades y centros de investigación para precisamente probar prototipos o algoritmos en proyectos de robótica, inteligencia artificial y visión por computador. La razón para usar este dispositivo en este tipo de proyectos es porque no es costoso en comparación con otros módulos y además es fácil de manejar pues existen drivers para conectarse con el AR.Drone directamente y enviarle comandos de velocidad o posicionamiento.

Así como se sabe, un cuadricóptero consta de cuatro palas del rotor. Y cada uno de sus rotores producen un cierto flujo de aire y, a su vez una fuerza de aceleración sobre el cuerpo de la cuadricóptero, y al darle poder de manera similar a los cuatro motores a una velocidad determinada, entonces este puede despegar (Parrot (2016)).

\section{II.2.5 Comportamiento de los Motores del AR. Drone 2.0}

Y si se logra que todos los cuatro motores juntos y de manera combinada compensan exactamente la gravedad de la tierra, el cuadricóptero se quedará en posición vertical en un mismo lugar como se puede apreciar en la Figura 1. 

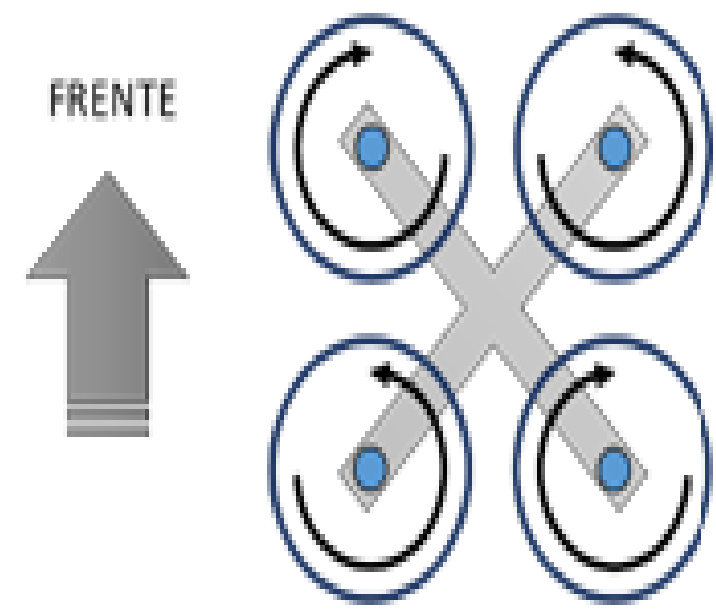

Figura 1: Motores de AR. Drone 2.0 a una misma velocidad sosteniéndole en una sola posición en el espacio (Sturm (2016)

Lo segundo muy importante es asegurarse de que los pares de la suma de los cuatro motores sea exactamente cero, es decir que si todos los cuatro rotores giraran en la misma dirección, entonces eso sería hacer girar el cuadricóptero alrededor del eje de vertical. Siempre se tienen dos motores montados opuestos en el mismo brazo que giran en la misma dirección, y los otros dos motores que giran en la dirección opuesta.

Y de esta manera, el par de los cuatro rotores suman a cero, mientras que el empuje compensa la gravedad de la tierra al generar sustentación.

Ahora para el ascenso, con sólo aumentar de manera equitativa la velocidad de los cuatro motores este hará un cambio en el equilibrio en la posición de estable, procediendo a ascender. Por otro lado, si se reduce la velocidad por igual de los cuatro motores, entonces el cuadricóptero descenderá, esta descripción es mostrada de manera gráfica por la Figura 2.

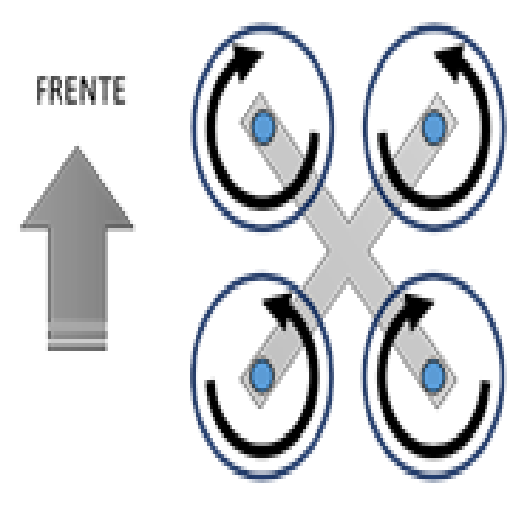

ASCENDER

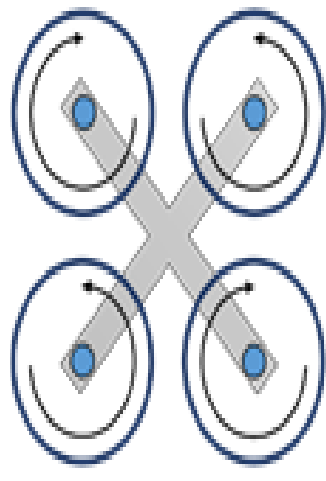

DESCENDEE

Figura 2: Motores de AR. Drone 2.0 en Ascenso y Descenso (Sturm (2016) 
Ahora bien, como ya he dicho, para mantener la orientación, es importante que los pares de los cuatro motores sumen exactamente a cero.

Sin embargo, también puede utilizar este principio para la inducción de una cierta rotación.

Por ejemplo, si usted quiere girar a la izquierda al cuadricóptero, se aumentaría la velocidad de los motores que giran en el sentido de las manecillas del reloj, ósea, los de giro derecho, que genera una tendencia de torsión que moverá al cuadricóptero a la izquierda.

Por otro lado, si desea girar a la derecha, entonces sería aumentar la velocidad de los que giran a la izquierda y decrecer la velocidad de los motores que rotan a la derecha. Por supuesto, si se desea mantener su posición vertical, el empuje total de los cuatro motores debe ser exactamente igual a la gravedad terrestre como se puede ver en la Figura 3.
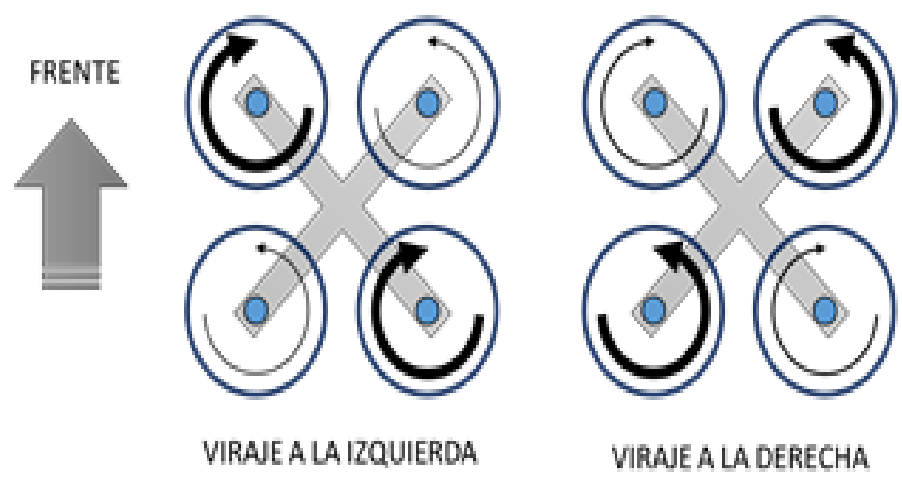

Figura 3: Motores de AR. Drone 2.0 y su comportamiento para virajes a la Izquierda y a la Derecha (Sturm (2016)

Ahora para mover hacia adelante (en la figura es la dirección hacia arriba) se debe reducir la velocidad de su motor delantero y aumentar la velocidad de su motor trasero.

Y de esta manera, la cuadricóptero se inclinará hacia delante ya que el empuje ya no está perfectamente alineado con la gravedad de la tierra, moviendo el centro de gravedad hacia adelante del eje, dando lugar a una aceleración, entonces, en la dirección de avance.

Del mismo modo, si se desea volar el cuadricóptero hacia atrás, se aumentaría la velocidad del motor delantero y se reduciría la velocidad del motor hacia atrás como lo demuestra gráficamente la Figura 4. 


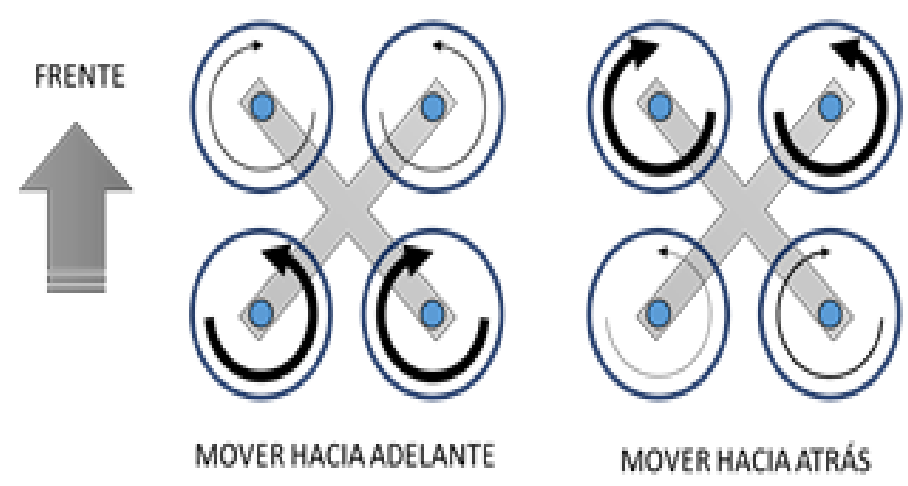

Figura 4: Motores de AR. Drone 2.0 y su comportamiento movimientos hacia Adelante y hacia Atrás (Sturm (2016)

Del mismo modo, también se puede mover hacia la izquierda y a la derecha basado en el mismo principio.

Y, por supuesto, también se puede combinar todos estos movimientos al mismo tiempo, por ejemplo para mover a la izquierda y al mismo tiempo girar a la izquierda para ascender y así otras configuraciones según se visualiza en lo mostrado gráficamente por la Figura 5.

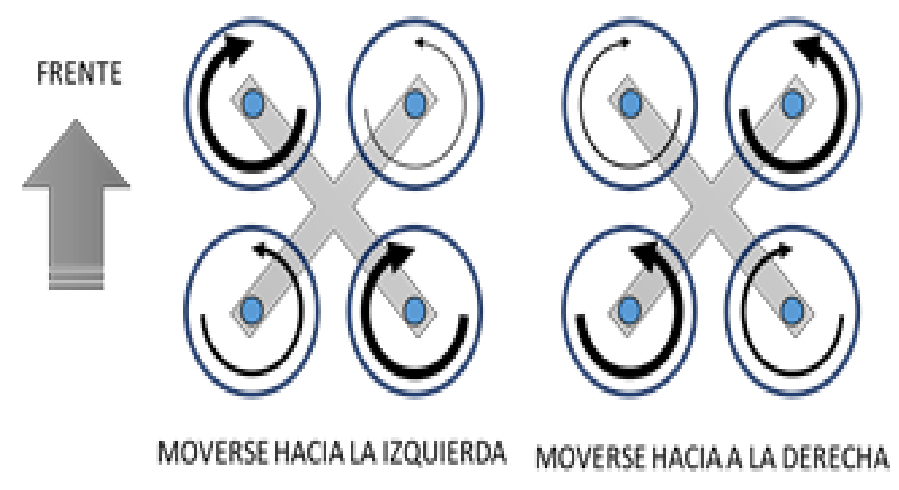

Figura 5: Motores de AR. Drone 2.0 y su comportamiento para virajes a la Izquierda y a la Derecha (Sturm (2016)

\section{II.2.6 Características del Parrot AR. Drone 2.0}

Este drone consiste en 4 motores, denominados motores sin escobillas.

Tienen un consumo de potencia nominal de 14.5 watts, por lo que la el cuadricóptero en general consume alrededor de 60 a 80 watts, con alguna pérdida.

Cada motor tiene su propio controlador que es dirigido por un pequeño CPU que controla la velocidad del motor.

Posee una batería de polímero de litio que brinda energía al Cuadricóptero de entre 10 a 15 minutos, está se encuentra en centro de la estructura cuadricóptero. 


\section{II.2.7 Los Sensores}

A continuación se muestran las características más técnicas.

1. El cuadricóptero tiene en su interior una unidad de medición inercial que consiste en un giróscopo de tres ejes, un acelerómetro de tres ejes, y una brújula, o un magnetómetro.

2. Posee también un sensor de ultrasonidos para medir la distancia al suelo y, además, un sensor de presión atmosférica que se pueden utilizar cuando el sensor de ultrasonido trabaja.

3. El sensor ultrasónico sólo tiene un rango de quizás más o menos tres metros.

4. Así que si se vuela más alto, especialmente al aire libre, el sensor ultrasónico no es muy útil, pero el sensor de presión atmosférica si, dando así la información de la altura del vuelo al drone.

5. También posee un sensor odométrico visual que en realidad es una cámara de ejecución a 60 cuadros por segundo, el cual es utilizado para el seguimiento de la posición visual del cuadricóptero.

6. Así mismo, el Parrot AR. Drone 2.0 posee una cámara frontal con resolución HD que también se puede acceder desde la computadora.

7. En el núcleo del Parrot Ardrone se encuentra un sistema completo para la ejecución de Linux, que consiste en un procesador en marcha del Cortex A8 de un gigahertz.

8. También cuenta con un adaptador de host USB 2.0 en donde se puede añadir memoria adicional para la grabación de vídeos, o un módulo GPS.

9. Posee igualmente un adaptador de red inalámbrica, con el que se puede comunicar con el cuadricóptero cuando está en el aire.

Una gran ventaja de este equipo y que lo hace tan atractivo para experimentación es que la interfaz de programación de aplicaciones, abreviada como API, por sus siglas en inglés, es una fuente totalmente abierta, por lo que es posible comunicarse con el cuadricóptero y obtener todos los datos de los sensores y enviar de vuelta comandos de desplazamiento al cuadricóptero.

\section{II.2.8 Programa Autoflight}

Autoflight es un programa de libre acceso, que se descarga del internet, y que puede ser modificado por el usuario. Muchas de sus ventajas es que puede ser ejecutado tanto en Windows como en Linux. Presenta a través de la pantalla de la computadora las capturas de imágenes hechas por la cámara del Parrot AR.Drone 2.0 y obtiene los datos de los sensores descritos en los párrafos anteriores, así como una vista del mapa y la secuencia de vídeo en tiempo real.

Su diseñador, Lukas Lao Beyer (Beyer (2016)) publica sus proyectos personales, siendo estos de código abierto, con el fin de que sirvan de base para nuevos proyectos, específicamente en programación y electrónica relacionada, y sirven sobre todo con fines autodidactas, al proporcionar documentación útil. 


\section{II.2.9 Autoflight: Autoscript}

Autoscript, es la plataforma para programación de Autoflight, en donde a través de él se pueden escribir scripts de Python para automatizar, v de una u otra manera, hacer autónomo el comportamiento del AR.Drone (Beyer (2016)).

Se caracteriza por el uso de funciones predefinidas que son utilizadas para controlar los movimientos, leer sensores, procesar información, entre otros. El mismo diseñador propone información sobre el cómo empezar a escribir guiones y descripciones detalladas de todas las funciones disponibles.

Al escribir secuencias de comandos que utilizan el poder de Python para controlar el Parrot AR.Drone 2.0, se puede lograr la programación de una ruta predefinida en pocos minutos o crear complejas aplicaciones de vuelo autónomo.

Igual brinda la probabilidad de un vuelo como un dispositivo de juego, y es que al conectar un mando de juegos a la computadora, se pueden configurar los controles y volar el drone de una manera más intuitiva. Autoflight también permite guardar los datos recopilados al brindar la oportunidad de exportar las lecturas del sensor en archivos CSV, legibles por la mayoría de las aplicaciones de hojas de cálculo.

\section{II.3 La Metodología}

El estudio presentado se considera exploratorio, dado que su objetivo es examinar un tema o problema de investigación poco estudiado o que no ha sido abordado antes. En el caso del estudio presentado la literatura consultada reveló que en Honduras, únicamente hay guías no investigadas e ideas vagamente relacionadas con el problema de estudio, de hecho no se encontraron trabajos de tesis de grado o posgrado que utilizaran el programa Autoflight para recoger evidencias experimentales para una investigación. Adicionalmente el abordaje propuesto establece una perspectiva relativamente nueva, la cual consiste en analizar la programación para volver autónomo a una aeronave no tripulada, con distintas finalidades entre las que se pueden mencionar aplicaciones en ambientes pedagógicos dentro o fuera de los tres niveles de la educación formal.

El valor agregado de esta investigación es que ayuda a familiarizarse con un fenómeno poco conocido, se obtiene información para realizar una investigación más completa de un contexto particular, investigar nuevos problemas, identificar conceptos o variables promisorias, establecer prioridades para investigaciones futuras, fundamentar empíricamente afirmaciones y postulados.

Existe un cierto conocimiento del funcionamiento y aplicación de un vehículo aéreo no tripulado; sin embargo, existen muchas características técnicas que deben ser analizadas previo la puesta en marcha de este vehículo de manera autónoma, lo cual es fundamental en esta investigación.

\section{II.3.1 Diseño Experimental}

La navegación aérea autónoma no requiere de ninguna manipulación de parte del ser humano durante la operación de vuelo; esta es la finalidad del presente proyecto de investigación: lograr que el cuadricóptero utilizado con la información recibida a través del sistema del programa Autoflight, en su apartado de Autoscript, en donde a través de lenguaje de programación Python se pueden generar directrices de manera secuencial, desarrollando de esta manera un 
vuelo automático en sus diferentes etapas, pudiendo completar este de manera independiente.

\section{II.3.2 Variables}

La presente investigación utilizará prevalentemente datos cualitativos, relacionados con secuencias y tipos de movimientos realizados por el vehículo aéreo no tripulado, la obtención de los datos para la manipulación de la variable se fundamentará en: el programa Autoflight, el lenguaje Python, el AR. Drone 2.0 y Windows 7 de 64 bits. Por todo lo anterior se definen:

- Variable dependiente: modificaciones la secuencia de comandos Python a ser utilizados en Autoscript del programa Autoflight de acuerdo a la precisión del drone en las prácticas realizadas respondiendo a órdenes establecidas a través de dichos comandos y,

- Variable independiente: Diseño y características del AR. Drone 2.0.

\section{II.3.3 Plan de Análisis}

Una vez concluidas las etapas de colección y procesamiento de datos en cada experimentación se examinará y analizará la interacción entre el programa de software Autoflight, el hardware o sistema operativo de la computadora y el vehículo aéreo no tripulado en este caso el AR. Drone 2.0. Conforme a los resultados obtenidos se harán ajustes al software con el fin de obtener la precisión requerida para lograr un vuelo autónomo.

Al finalizar la investigación se podrán desarrollar actividades en un ambiente no controlado limitado por la autonomía intrínseca de la aeronave y el alcance de la señal entre el drone y la computadora.

\section{RESULTADOS}

\section{III.0.1 Los Expertos}

En Honduras es limitada la cantidad de experto en diseño y programación de vehículos aéreos no tripulados.

De los que se conoció durante esta investigación fue a los señores Allister Stefan, quien se dedica de manera aficionada a esta disciplina, pero que posee cursos oficiales en el extranjero, y al señor León Rojas, quien se desempeña como asesor en la Universidad de Defensa de Honduras.

La entrevista fue realizada al Señor Allister Stefan, en donde brindo una asesoría acerca del funcionamiento de dichos aparatos.

Dentro de los datos de mayor relevancia proporcionados por el están los siguientes:

1. Hace referencia que los alcances en diseño y programación por parte del país han nacido a nivel de la afición del aeromodelismo. 
2. Que en el país existe el potencial para promover proyectos que pueden ser orientados en aplicaciones civiles con el fin de solucionar que afectan el bienestar social, entre estas áreas, están las agrícolas, protección del medio ambiente, apoyo durante desastre naturales, estudios de minería, entre otros.

3. Que existe poco o nada de legislación para la operación de aeronaves no tripuladas en el país, lo que no va a tono con las tendencias mundiales.

4. Que existe una voluntad de parte de la comunidad de aeromodelismo a apoyar proyectos propios del área de operación, diseño y programación de drones.

\section{III.0.2 Apoyo Técnico con el AR. Drone 2.0}

Parte de la instrucción recibida fue la reparación del sistema de propulsión del drone, ya que en ensayos iniciales, sin la presencia del experto, fue dañado al impactar con paredes y piso. En esos impactos fueron doblados los ejes de las hélices, desgastados y rotos algunos dientes de engranajes de transmisión de rotación y erosionados los bordes de ataque de las palas de estas, provocando que la sustentación fuera desequilibrada, tendiendo que el drone se desplazara lateral o frontalmente, cuando su posición debería ser estable y nivelada.

Es por esta razón, que se inició con un reconocimiento del equipo adquirido para la investigación, en donde fueron reemplazados dichos ejes, engranajes y hélices por otras que el Doctor Stefan tenía en su taller. En ese procedimiento se mostró que cada hélice posee una letra acorde a su posición, así como la forma de remover el seguro que sostiene la hélice con el engranaje y el acople a través del eje. En la Figura 6 se ve la secuencia del procedimiento de reemplazo de piezas.
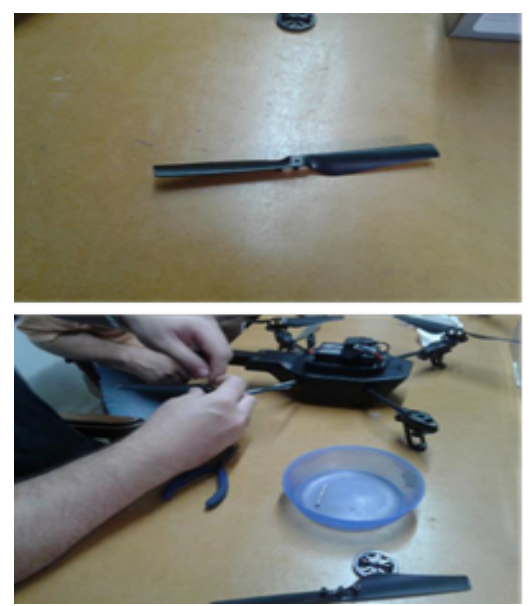
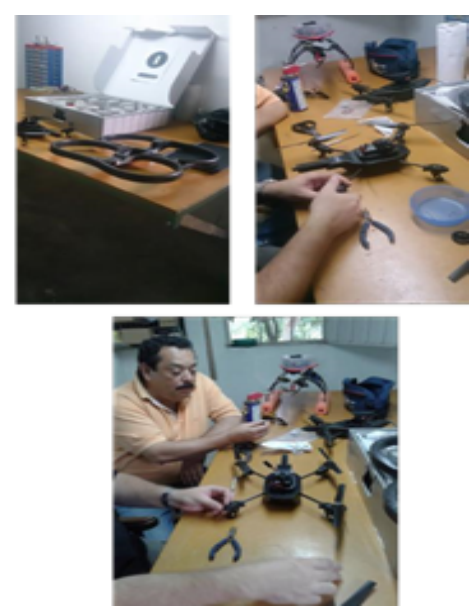

Figura 6: Procedimiento de Reemplazo de Hélices, Engranajes y Ejes del AR. Drone 2.0 (elaboración propia).

Posteriormente se procedió a la puesta en vuelo del drone a través de una aplicación instalada en la Tablet, y dando seguimiento al siguiente procedimiento:

1. Conectar la batería del aparato. 
2. El AR. Drone 2.0 hará un test de motores, los que son acompañados por un característico sonido fino y un movimiento de la hélice de manera secuencial.

3. Cuando lo anterior ha sucedido, el drone crea una red inalámbrica (WiFi) en su entorno.

4. Seguidamente se activa el sistema de señal inalámbrica de la Tablet (o Smartphone) y se detecta el término "ardrone2 _.....", como una fuente de Información Inalámbrica.

5. Se procede a activar la aplicación AR.FreeFlight 2.4 la cual es la estándar, pero existen otras más.

6. Presionamos el botón TAKE OFF, en donde aparecerá la imagen en tiempo real, la cual es percibida a través de la cámara frontal del aparato, también sale información adicional como,
a. Nivel de Batería
b. Botón de Configuración
c. Red Inalámbrica (WiFi)
d. Indicador de Grabación
e. Pulsador para tomar fotografías

f. Botón de Emergencia (no recomendable si está volando a gran altura, pues esto provocaría el apagado inmediato, por lo que se desplomaría sin control).

7. Se procede a hacer el ajuste horizontal (Flat Trim), lo cual es recomendable hacerlo en el primer vuelo, para que el aparato se calibre, poniendo al drone en un lugar plano y nivelado.

8. Es recomendable iniciar el despegue sobre una imagen (la cual viene una sugerida dentro de la caja del drone), controla la altura a través de un sensor de ultrasonido, y la posición la controla a través de una cámara cenital que se mantiene viendo la imagen bajo de él.

\section{III.1 Practicas Realizadas con el Simulador del Curso en Línea Na- vigation for Flying Robots}

Uno de los procesos que apoyó de manera robusta a esta investigación fue una serie de prácticas haciendo uso de un programa ofrecidos a través del Curso en Línea "Navigation for Flying Robots", el que en realidad, es la parte más interesante ya que los ejercicios prácticos de programación son desarrollados en lenguaje Python, lenguaje también utilizado por el programa Autoflight.

Este simulador desarrollado está basado en la web, específicamente para un cuadricóptero, y esto significa que se pueden implementar inmediatamente cualquier nueva configuración que se desee ensayar. Se tomó especial cuidado de que en realidad el código que se escribiera también fuera ejecutable en un Parrot AR.Drone. Un ejemplo de cómo se introducen los códigos para el simulador se pueden observar en la Figura 7. 


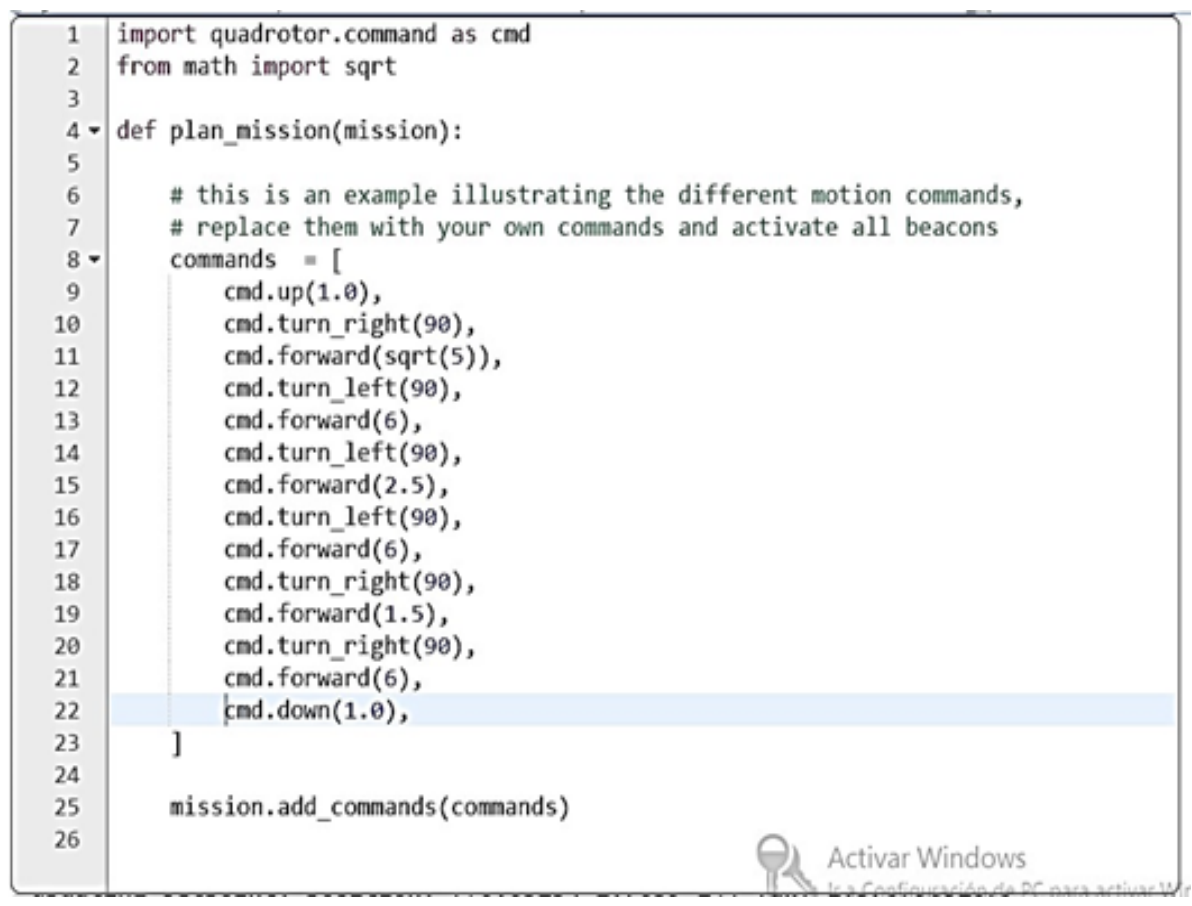

Figura 7: Códigos aplicados como instrucción al Drone en el Ejercicio Descrito (elaboración propia).

Así que si los ensayos en el simulador también podían ser demostrados en un Parrot AR.Drone, probando posibles soluciones antes de hacerlos de manera real.

A continuación se plasman uno de los ensayos realizados y su solución a través de un lenguaje de programación y algunos de los gráficos que muestran su realización. Esta actividad consistió en enviar al cuadricóptero a lo largo de la trayectoria alrededor de las balizas, haciendo que estas fueran tocadas, con el fin de que su color fuera de ROJO a VERDE.

Cada cuadrado de la rejilla tiene una longitud lateral de 0.5 metros. Para enviar el cuadricóptero a lo largo de su trayectoria se dio clic en el botón de reproducción, como se puede apreciar en la Figura 8. 

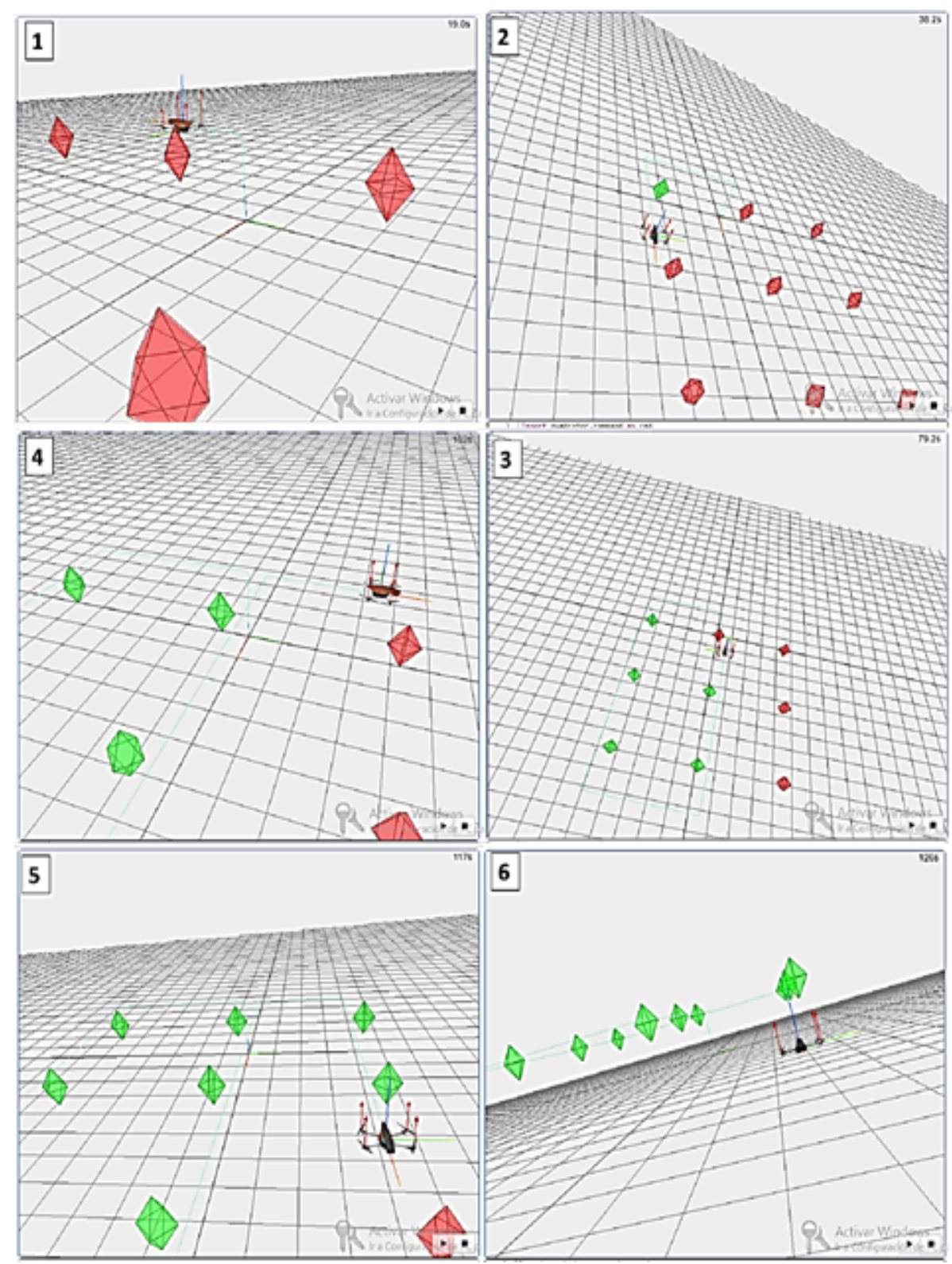

Figura 8: Imágenes del Simulador del Drone en el Ejercicio Descrito (elaboración propia).

\section{III.2 Practicas Realizadas con el AR. Drone 2.0 y Autoflight}

El proceso realizado durante las actividades anteriores, desarrollaron el soporte suficiente para dar el siguiente paso que es de la experimentación con el equipo AR. Drone 2.0.

Las actividades realizadas fueron de simple comprobación, ya que lo que se pretendía era la vinculación entre el AR. Drone 2.0 y la computadora, a través del 
interfaz que utiliza señal inalámbrica entre ambos. A continuación se presenta una de las prácticas realizadas en donde se pretende que el Drone realice lo siguiente:

\section{Despegue}

2. Se mantenga estable durante 5.5 segundos en vuelo estable

3. Avance a una velocidad de 1 metro por segundo durante un segundo

4. Se mantenga estable durante 5.5 segundo en vuelo estable

5. Vire noventa grados $\left(90^{\circ}\right)$ hacia la derecha.

6. Vuele hacia adelante a una velocidad de 1.0 metros por segundo

7. Se mantenga estable durante 5.5 segundos en vuelo estable

8. Vire noventa grados $\left(90^{\circ}\right)$ hacia la derecha.

9. Vuele hacia adelante a una velocidad de 1.0 metros por segundo

10. Se mantenga estable durante 5.5 segundos en vuelo estable

11. Vire noventa grados $\left(90^{\circ}\right)$ hacia la derecha.

12. Vuele hacia adelante a una velocidad de 1.0 metros por segundo

13. Se mantenga estable durante 5.5 segundos en vuelo estable

14. Vuele hacia adelante a una velocidad de 1.0 metros por segundo

15. Aterrice

En la Figura 9 se pueden ver un ejemplo de cómo se ingresaron los comandos al programa AutoFlight para su programación.

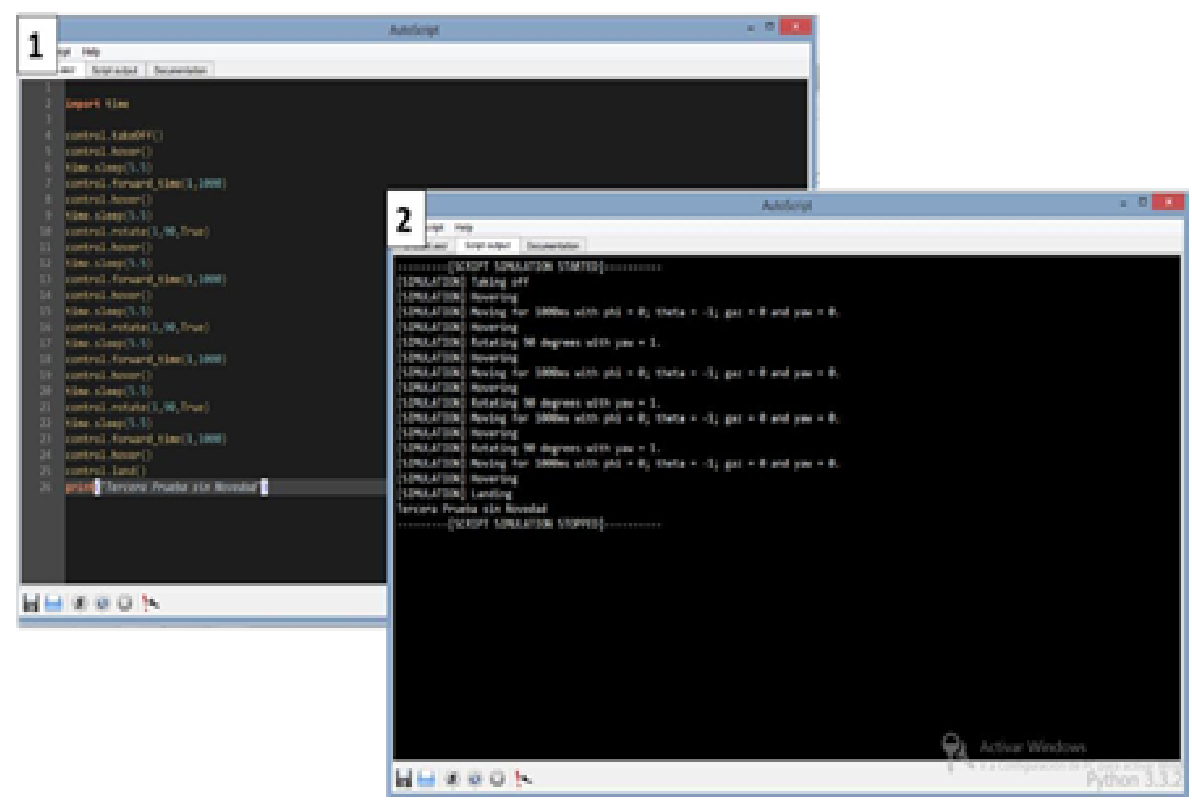

Figura 9: Activación de la programación en AutoFlight diseñada para la práctica descrita (elaboración propia)

El vuelo fue realizado sin ninguna novedad, haciendo el Drone lo solicitado a través de Autoscript del programa Autoflight. 


\section{DISCUSIÓN}

Se examinaron dos formas en las que se puede manipular un vehículo aéreo no tripulado, por el manejo simultáneo al movimiento del artefacto, mediante un control remoto y mediante la previa programación de sus movimientos; la primera forma es basada en una Aplicación para Teléfonos Inteligentes o Tablet, la cual fue desarrollada por el fabricante del Drone, lo que ayudo a entender el comportamiento de la aeronave, sus limitaciones y ventajas; la segunda brindo la oportunidad de programar desde una computadora portátil o de escritorio las acciones que el Drone debía realizar, pudiendo presentar una gran extensión de oportunidades, que se limita únicamente por la imaginación del usuario y las características del equipo, pero que propone una excelente plataforma de experimentación para nuevas aplicaciones. En cualquier caso con las dos formas de manejo se pueden examinar los aspectos mencionados en el párrafo anterior, será la experiencia la que nos indique cuál de las dos formas de manipulación se revela más adecuada para la labor investigativa del Departamento de Ciencias Aeronáuticas en dinámicas de investigación e innovación intradisciplinarias, interdisciplinarias o transdisciplinarias. Este tipo de prácticas vienen a fortalecer los sistemas educativos, ya que el software y hardware de códigos abiertos proponen un nuevo campo de investigación e innovación, en el cual el alumno podrá realizar experiencias que no necesariamente están escritos en un manual de laboratorio, ya que las opciones son infinitas.

\section{CONCLUSIÓN}

1. Razones relacionadas con la disminución en los tiempos de operación, los ahorros ostensibles en los costos de los vuelos no tripulados en comparación con vuelos tripulados, la versatilidad en la amplia gama de operaciones, por mencionar solo algunos ejemplos, convierten a los vehículos aéreos no tripulados comúnmente conocidos como Drones en una opción por lo que cada vez más personas tendrán una preferencia; dado este hecho la UNAH deviene cada vez más obligada a ocuparse de estudiar todos los aspectos relacionados con este tipo de aeronaves, incluyendo entre otros sus estructuras, su desempeño, su programación y sus equipamientos..

2. En este primer acercamiento el DCA ha examinado un tipo de vehículo no tripulado con un interfaz que permite la programación de sus movimientos a través de lenguaje de programación computacional, básicamente en condición de programador de software, como es de esperarse, quedan pendientes muchos aspectos a explorar entre los que se pueden mencionar las aplicaciones que involucren el uso de los sensores que el AR. Drone 2.0 posee, su sistema de navegación satelital, así como áreas más amplias como la definición precisa de las ventajas en términos económicos y de precisión de los datos, el uso de este tipo de vehículos para la innovación educativa, el desarrollo de proyectos de vinculación universidad-sociedad mostrando los alcances de los vehículos no tripulados para divulgar masivamente el fenómeno de la aviación, entre otros.

3. Luego de esta primera aproximación en la que el DCA asumió el rol del usuario de las funciones básicas de uno de los muchos tipos de vehículos no tripulados existentes, se avizoran en el horizonte al menos dos grandes etapas de la investigación sobre el tema: una segunda etapa sería la exploración en aplicaciones especializadas para los campos de las ciencias espaciales y para los demás campos de la ciencia en los que la UNAH está generando nuevos conocimientos y, una tercera etapa puede ser la generación de innovaciones en la construcción, programación y equipamiento 
de vehículos no tripulados para aplicaciones en los ámbitos académico, comercial, productivo y de recreación.

4. Considerando que los vehículos no tripulados están teniendo un gran impulso desde la perspectiva tecnológica, personas, organizaciones e instituciones que en Honduras se han dedicado intensivamente al tema como lo han hecho los Señores Allister Stefan y León Rojas, serán referencia obligada para consultar los distintos aspectos relacionados.

\section{Agradecimientos}

Al Doctor Allister Stefan por sus valiosos aportes en información y orientación tecnológica sobre aeronaves no tripuladas que fueron soporte esencial en el diseño y redacción de esta investigación.

\section{Referencias}

Beyer, L. L. (2016). Pc control software for the parrot ar.drone 2.0 and bebop uavs. AutoFlight. Descargado de http://electronics.kitchen/autoflight

FENERCOM. (2015). Los drones y sus aplicaciones a la ingeniería civil. Madrid: Gráficas Arias Montano, S.A.

Ministerio de Defensa de España, E. (2009). Uas unmanned aircraft system sobre su integración en el espacio aéreo no segregado. Madrid: Imprenta del Ministerio de Defensa.

Parrot, S. (2016). Parrot, ar. drone 2.0. Descargado de http://www . parrot .com/usa/products/ ardrone-2/

Sturm, $\mathrm{j}$. (ZUंठ́). Autonomous navigation for flving robots. Computer Science. Descargadn de ht.tps://www.edx.org/course/autonomous-navigation-flying-robots-tumx -autonavx-0 